\title{
Thermo Harvesting - More and Enduring Power for Wireless Systems
}

\author{
Burkhard Habbe \\ Micropelt $\mathrm{GmbH}$ \\ Emmy-Noether-Str. 2 \\ 79110 Freiburg Germany \\ www.micropelt.com
}

Wireless sensor systems gain increasing market shares in a growing number of markets, from smart metering into Industrial condition and process monitoring. The driving force of almost all of these nodes is some sort of a battery. Hence, battery capacity is predominantly determining performance and features of such systems. Both of these criteria as well as the system's duty cycle have to follow the limits given by capacity and price of the energy storage. As battery changes - except for private households - cause additional maintenance cost and recycling issues, many potential users avoid wireless devices altogether.

On the other hand the power requirements of novel radio and sensor systems have decreased substantially, making it possible to drive e.g. smart energy meters for remote readout beyond their calibration period of 10 years - with just a button cell. Sure, the trade-off is in a reduced feature set and the least possible duty cycle to ensure both regulatory compliance and basic functionality requirements of the energy provider. Many useful and advanced features such as daily readout, peak usage billing and usage control are renounced due to a lack of installed energy.

However, with closer proximity of applications to condition or process monitoring in industrial environments both minimum required feature-set and duty cycle expand considerably. Reliability is

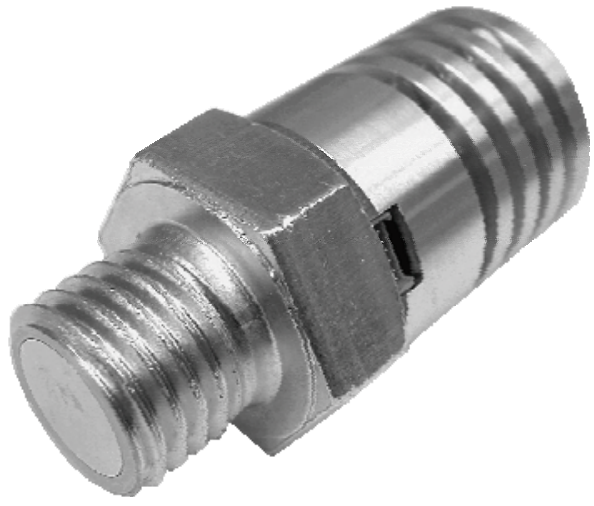

Fig. 1: Bolt with integrated Thermoharvester indispensable and decreasing the scan rate may compromise the application. Wireless transmission must be as reliable as its wired equivalent, despite multiple sources of electromagnetic interference along the transmission path. Wireless sensors have the capability of removing guesswork and indirect risk management from the agendas of both process operators and maintenance managers. Statistical and simulation based maintenance and operation may be upgraded to real control, enhancing the utilization of assets while reducing operational risks. However, a major factor standing in the way of wireless sensors remains the maintenance cost and effort associated with batteries.

To overcome both the constraints of limited power capacity and extensive maintenance cost of batteries a growing number of vendors offer a variety of energy harvesting technologies and devices. The concept they all have in common is converting excess or waste energies such as vibration, motion, magnetic and electric fields, and heat into a useful form of electrical energy. These offerings converge well with the decreasing power requirements of wireless systems and microelectronics in general.

Micropelt, a Freiburg, Germany based specialist in thinfilm thermoelectrics has a clear focus on harvesting energy from excess heat which could be generated by

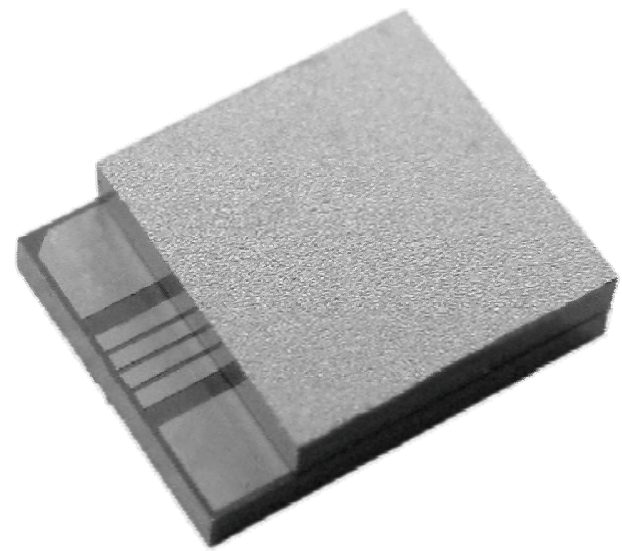

Fig. 2:Micropelt MPG-D751 Thermogenerator chip. 
hot processes, friction in all kinds of bearings, energy production and transport, combustion and many more. The fundamental element of Micropelt's technology is their patented combination of advanced thermoelectric material science and chip making technologies.

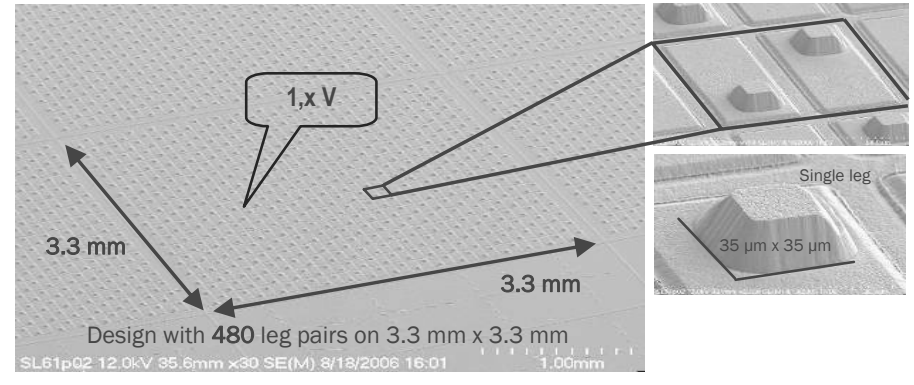

Fig. 3: Micro-structured Bismut-Telluride is the core element of thermogenerators.

The first allows for producing a unique thin film of thermoelectric BismutTelluride $\left(\mathrm{Be}_{2} \mathrm{Te}_{3}\right)$ while the latter provides a means for micro-structuring this thin film in a MEMS-like process and produce chip-sized devices with the economies-of-scale of conventional microelectronics. Bonding together corresponding parts of two wafers of differently doped ( $p$ or $n$ ) thin films eventually generates one or multiple thermoelectric devices in only one process step. Shaping and scaling the minute TE Elements (TE legs) with smallest dimensions around 35 microns in length and height allows for adapting devices for specific purposes including thermal harvesting, device cooling and thermal process control at and near chip level. Infineon Technologies initiated the development of this technology in 1998 by awarding a related R\&D contract to the Freiburg based Fraunhofer IPM. Micropelt GmbH was founded in early 2006 after a VC-leveraged management and team buyout.

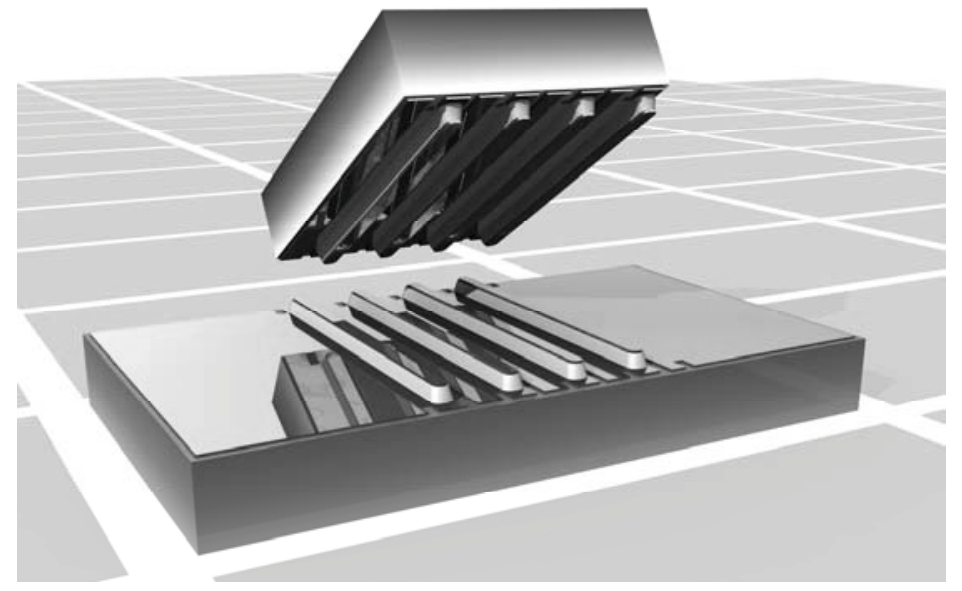

Fig. 4: Thin film thermoelectric element before bonding $\mathrm{p}$ and $\mathrm{n}$ parts together
One particular advantage of Micropelt's TE legs being 10 to 100 times smaller than their conventional counterparts is associated with the Seebeck law of thermo generation. According to this law each thermocouple or $\mathrm{p} / \mathrm{n}$-doped leg pair generates a specific voltage per Kelvin of temperature gradient applied. This device related constant is usually found in the low microvolt range. There are only two known ways for obtaining voltages from thermoelectric devices, which are useful for driving electronic devices: One is through low-level step-up conversion of the voltage of few TE leg pairs. This option takes more than $50 \%$ of the input power for the conversion itself. The other option is implemented by Micropelt: Increasing the number of TE leg pairs to several hundreds yields useful voltage levels directly from the device. The footprint of a typical Micropelt thermogenerator, which contains around 480 leg pairs, is less than $15 \mathrm{~mm}^{2}$. With $10 \mathrm{Kelvin}(\mathrm{K})$ of temperature difference applied to this device it produces $1.4 \mathrm{~V}$ open circuit voltage and about $2 \mathrm{~mW}$ power at matched loads. Hence, this type of micro-thermogenerator lends itself much more towards compact mechanical and electronic integration than any conventional TE device.

Needless to say, that a power supply at this level has a very limited scope of addressable applications. However, energy storage and intelligent power management can change the picture indirectly as much as high temperature gradients could do directly. Let's see how this picture develops. 
At first a few hundred millivolts provided by the thermogenerator are to be stepped up by a DC-DC converter. Micropelt provides a specially designed solution. The current third device generation starts at low $300 \mathrm{mV}$ of input voltage, equal to $2.2 \mathrm{~K} \mathrm{dT}$. $1.2 \mathrm{~V}$ to $1.8 \mathrm{~V}$ open circuit voltage are available at this point. Best efficiency of $74 \%$ is reached from $0.5 \mathrm{~mW}$ of input power, equal to $5.3 \mathrm{~K}$ net dT across the thermogenerator (TEG). To get $3.3 \mathrm{~V}$ at the output $520 \mathrm{mV}$ input voltage are required, equal to only $3.7 \mathrm{~K}$ net $\Delta \mathrm{T}$.

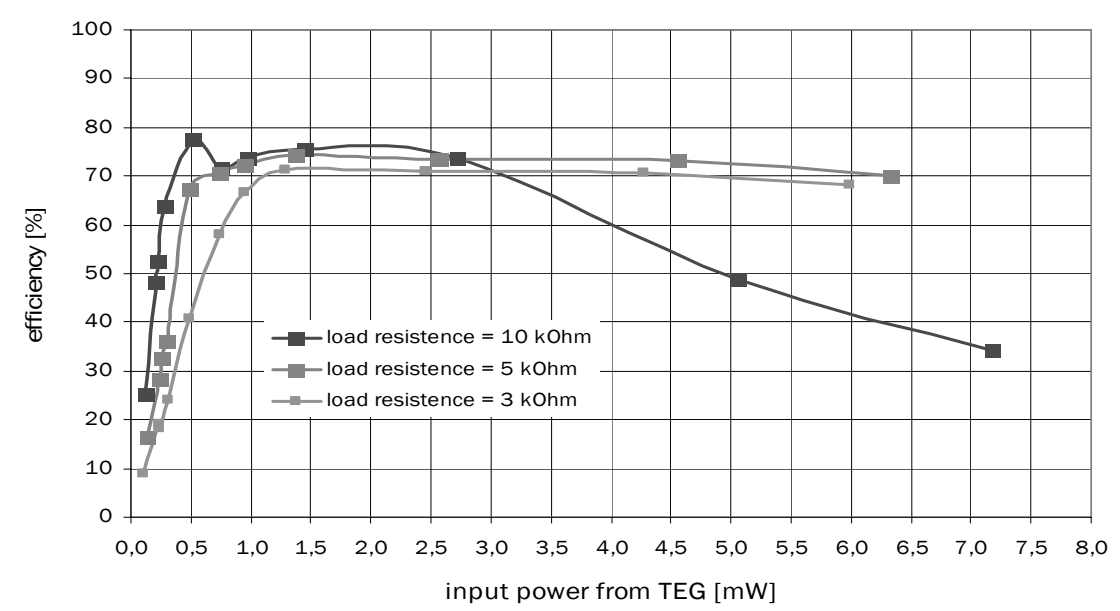

Fig. 5: Efficiency diagram of DC-DC converter for thermogenerator MPG-D751

For a further completing the picture it is useful to consider the energy balance of the harvester versus the duty cycle needs of the application. Only with a positive balance an energy harvesting driven system can operate without external power supply.

The stand-by power consumption of the attached system is taken directly from the harvesting result. Functions to be sustained may include clock generator, wake-up timer, heartbeat, wake-on-signal functions and charge and power management. The power consumed by these functions is not available for the actual duty cycle. Any power supplied by the thermogenerator beyond stand-by, let's call it duty cycle budget (DCB), must be collected for driving the actual duty cycle which may require a multiple of the incoming current.

\section{calculated matched output power vs.DT}

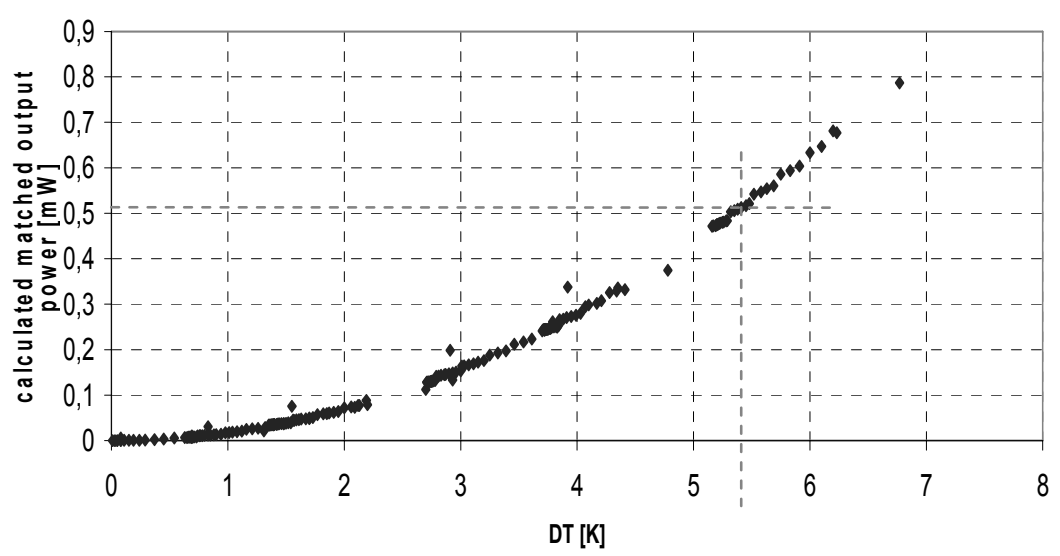

Fig. 6: Performance diagram of standard thermogenerator MPG-D751
To support high current duty cycles an energy storage must be provided, e.g. a capacitor, a gold cap or a suitable type of rechargeable battery. Desirable properties of that storage include low charge current to maximize harvesting results; low self discharge and flat discharge characteristics to ensure high power availability during no-charge periods, and of course high temperature stability for the same reason.

For optimal energy and charge efficiency on one hand and best support of the intended system functionality an intelligent energy management is indispensable. Essential functions are to ensure

maximal charge rates at each operating condition, prevent destructive deep discharge of rechargeables, control power budgeting, and provide energy status information. Any particular implementation, however, must be matched with the requirements of the attached wireless system.

Due to the limited capacity of any battery and an absolute need for maximal battery life at minimal size and cost, systems are often not designed for best performance and user benefit. Desirable yet energy 
consuming functions are omitted, even more so as architecture, topology and scan rates of the associated wireless sensor network may dictate extensive operating power consumption. However complex the system specification, at some point the topic of power requirements will be fully specified. Now we need to determine whether the wireless node should be using primary batteries or a thermo harvester as a power supply.

Batteries are cheap and easy to implement, but the issues associated with their maintenance and disposal prevent many potential users of wireless systems from actual implementation. Market studies identify this concern as a major inhibitor of wireless sensor networks with $70 \%$ of the interested industry being sceptic about batteries (OnWorld). Let's take a look at a theoretic application and find out whether it is possible to run it without batteries, wires and maintenance:

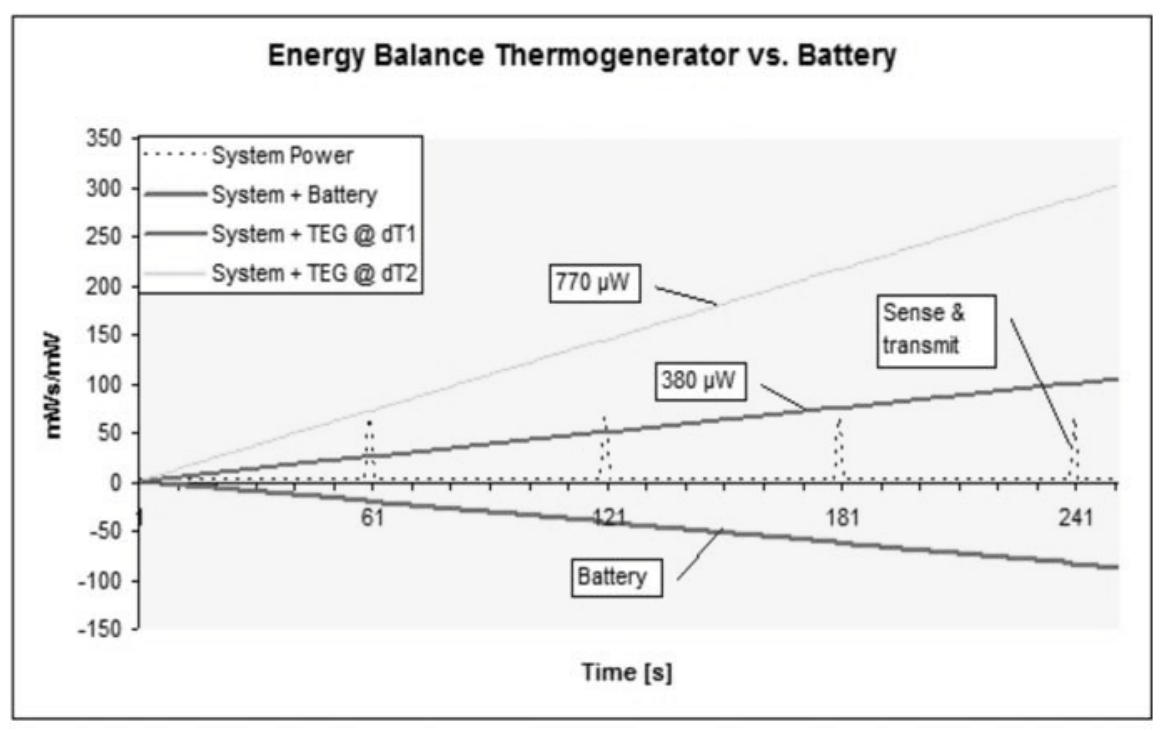

Fig. 7: Any positive energy balance provides additional power over batteries.
The system runs at $3.3 \mathrm{~V}$ and draws $100 \mu \mathrm{A}$ of current in sleep mode, resulting in stand-by power of $330 \mu \mathrm{W}$. The duty pulls $20 \mathrm{~mA}$ from an energy storage, e.g. a thin film battery. Duty cycle is defined as one transmission per minute, each taking 20 milliseconds. This amounts to $3.3 \mathrm{~V} * 0.02 \mathrm{~A} * 0.02 \mathrm{sec}$ $=1.32 \mathrm{mWsec}$ of power beyond stand-by. This is the minimum DCB the thermo harvester has to supply beyond $330 \mathrm{~mW}$ sleep load. The available harvesting time is $60 \mathrm{sec}-$ $0.02 \mathrm{sec}=59,98 \mathrm{sec}$. Consequently it takes 1.32 $\mathrm{mWsec} / 59,98 \mathrm{sec}=$

$0.022 \mathrm{~mW}=22 \mu \mathrm{W}$ of excess power to feed the duty cycle from the energy harvested and stored during stand-by. The minimum total harvesting power before efficiency loss is now determined as $352 \mu \mathrm{W}$. Assuming a charge efficiency of $80 \%$ the required gross harvesting result increases to $22 \mu \mathrm{W} / 0,8=27,5$ $\mu \mathrm{W}$, demanding a total of $357,5 \mu \mathrm{W}$ of continuous power from the DC-CD converter attached to the thermo harvester. At this operating point, the conversion efficiency is in a steep rise towards a maximum $74 \%$. 50\% efficiency are taken from the related diagram, which calls for an approximate gross harvesting output of $700 \mu \mathrm{W}$. The required effective (net) temperature gradient across the thermogenerator is taken from the respective performance diagram. From 6.3 Kelvin (K) the MPG-D751 thermo generator chip produces the specified power. The net result at the DC-DC converter output when summarized over one year of constant operation amounts to $2,600 \mathrm{mAh}$. This is the available budget for the considered wireless system. For three years of operation on one battery pack one would have to install almost $8 \mathrm{Ah}$ of battery capacity. As for the energy harvesting power supply, this is just the minimum available power. Any increase in the temperature gradient would straight in a corresponding surplus in available power - for more duty cycles or an enhanced feature set of the attached system.

To finally make things complete in terms of thermal harvesting, it is inevitable to take a closer look at heat transfer to ambient air. This transfer is effected by some form of heat sink, which is dimensioned by the maximal permissible thermal resistance to achieve a desired heat flux. The known factors to consider first are the temperature gradient across the thermo generator and its thermal resistance. We find the heat flux after calculating $6.3 \mathrm{~K} * 12.5 \mathrm{~K} / \mathrm{W}=0.504 \mathrm{~W}$.

This heat flux passes all components and interfaces of the implemented thermal path, i.e. heat source surface adapter and TEG input adapter on the input side, TEG itself, and heat sink on the output side. 
The input side is conservatively assumed to have $1 \mathrm{~K} / \mathrm{W}$ of thermal resistance, which results in a gradient of $0.504 \mathrm{~K}$ across these components and their associated interfaces. As we are free to choose any heat sink we select one with a thermal resistance of $10 \mathrm{~K} / \mathrm{W}$. The temperature gradient of the heat sink when exposed to the known heat flux is determined as $10 \mathrm{~K} / \mathrm{W}{ }^{*} 0.504 \mathrm{~W}=5.04 \mathrm{~K}$. As voltages across electrical resistances connected in series added give the total supply source voltage, temperature gradients across the various parts of a thermal path add up to the total gradient across the system. In our example we find the total gradient as $0.504 \mathrm{~K}+6.3 \mathrm{~K}+5.04 \mathrm{~K}=11.844 \mathrm{~K}$. This means our system can operate between e.g. $25^{\circ} \mathrm{C}$ of ambient air and a warm surface of $37^{\circ} \mathrm{C}$ or more. Mounted on a heat source of $70^{\circ} \mathrm{C}$ the heat sink would need not more than $58^{\circ} \mathrm{C}$ of ambient air temperature. In fact, the result at this temperature level would even be better, as the thermoelectric performance index of BismuthTelluride increases until $140^{\circ} \mathrm{C}$ approx.

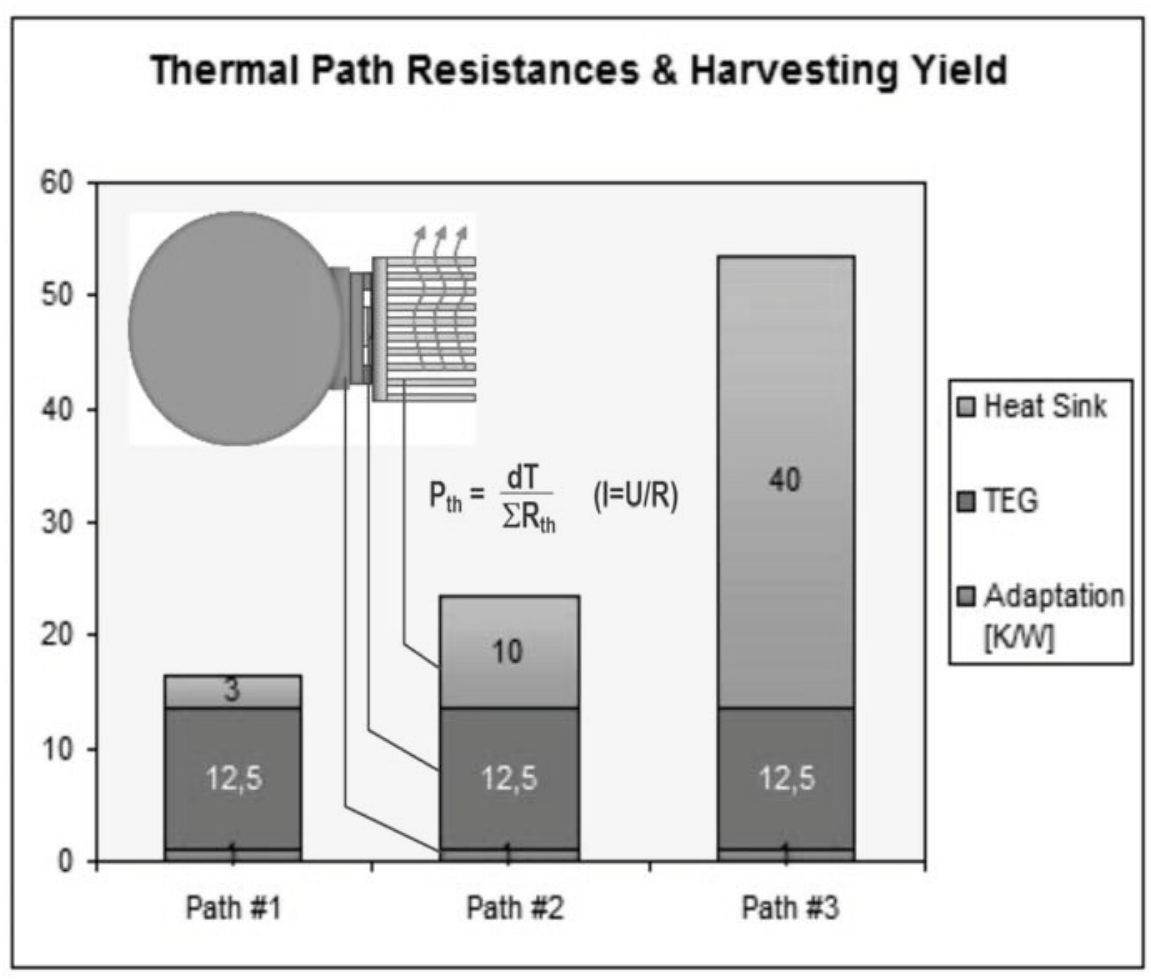

Fig. 8: Harvesting yield largely depends on heat sink efficiency
Due to design, cost or envelope constraints one might be forced to use a smaller, hence less efficient heat sink of e.g. $40 \mathrm{~K} / \mathrm{W}$ of thermal resistance. Applying the same math as before we find a temperature gradient of $20.16 \mathrm{~K}$ across this heat sink The result is a total or gross gradient of $26.994 \mathrm{~K}$. The previous $37^{\circ} \mathrm{C}$ on the heat source now need to be increased to almost $52^{\circ} \mathrm{C}$ to harvest the same amount of power. If we apply the first configuration to this gross thermal gradient we find from the respective performance diagram a gross harvesting power of more than 3.4 $\mathrm{mW}$. After DC conversion, now at full efficiency of $74 \%$ we dispose of an abundance of energy of $2.54 \mathrm{~mW}$. When harvested

throughout one year this energy amounts to an impressive $18.000 \mathrm{mAh}$. An attached system could be dimensioned for this annual energy consumption.

Obviously, thermoharvesting is more than just a somewhat complex workaround of batteries. Many wireless systems including wireless sensors can benefit through bigger power budgets and wired power supply. Feature sets of wireless devices may be enhanced as well as duty cycles can be increased. Huge amounts of toxic waste can be avoided and more system and maintenance intelligence can help reducing material cost and staff utilization. Thermo harvesters do not need to be integral parts of their sensor node. Separate gross or net harvesters may be deployed on any suitable heat source within a few meters of wired reach of the wireless sensor node. Power management may be located on either one side or the other, whatever works best. Supply

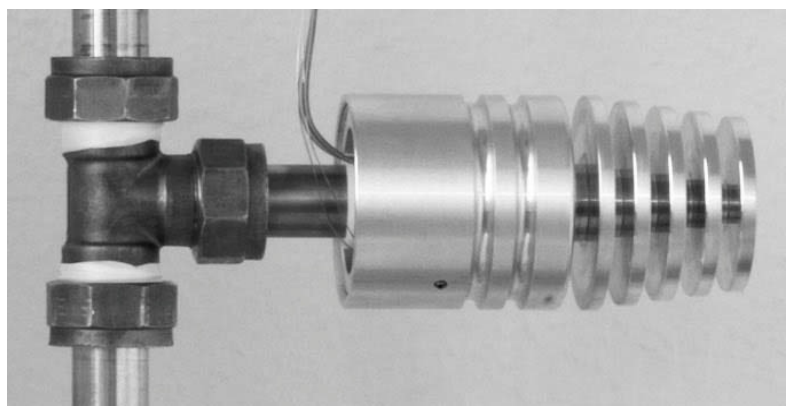

Fig. 9: TE Power PROBE Thermoharvester for natural convection 
performance of high voltage micro thermo harvesters as offered by Micropelt have converged with power requirements of modern wireless sensor devices to form a substantial contribution to boosting the acceptance and penetration of wireless technologies in multiple target markets. Finally, if a harvester appears too expensive over a few button cells - the first replacement service will pay the difference.

\begin{tabular}{|l|l|l|l|}
\hline Mode & $\begin{array}{l}\text { Time } \\
{[\mathrm{s}]}\end{array}$ & $\begin{array}{l}\text { Power } \\
{[\mathrm{mW}]}\end{array}$ & $\begin{array}{l}\text { Energy } \\
{[\mathrm{mWs}]}\end{array}$ \\
\hline Standby & $59,998 \mathrm{~s}$ & 0,0045 & 0,27 \\
\hline $\begin{array}{l}\text { Duty } \\
\text { Processor }\end{array}$ & 0,0005 & 18 & 0,027 \\
\hline $\begin{array}{l}\text { Duty } \\
\text { Radio }\end{array}$ & 0,0015 & 45 & 0,023 \\
\hline $\begin{array}{l}\text { Harvester-Continuous Power } \\
1 \mathrm{x} \text { Tx/min }=5,5(\mu \mathrm{W}) \\
10 \mathrm{x} \text { Tx/min }=14,8(\mu \mathrm{W})\end{array}$ \\
\hline $\begin{array}{l}\text { Fig. 10: Power budget for Ultra Low Power device } \\
\text { (Example) }\end{array}$ \\
\hline
\end{tabular}

